\title{
Enhancement of the Interlayer Interaction between Polystyrene and Polyvinyl Alcohol by Ozone Treatment
}

\author{
Lin Su, Jie Li, Jing Li, Qiaomei Chu, Bo Li, and Yiyang Liu \\ Research Center of Laser Fusion, China Academy of Engineering Physics, Mianyang 621900, China \\ Correspondence should be addressed to Yiyang Liu; cqmhb821@163.com
}

Received 28 October 2018; Accepted 21 April 2019; Published 18 June 2019

Academic Editor: Katja Loos

Copyright (c) 2019 Lin Su et al. This is an open access article distributed under the Creative Commons Attribution License, which permits unrestricted use, distribution, and reproduction in any medium, provided the original work is properly cited.

\begin{abstract}
The ozonization modification of polystyrene (PS) was conducted in acidic condition to enhance the interfacial interaction between PS and polyvinyl alcohol (PVA). Infrared spectra results revealed that the carbonyl and hydroxyl groups were successfully introduced to the PS film by ozonization. The hydrophobic PS was turned to be hydrophilic to some extent by contact angle experiment, which led to the enhancement of interfacial adhesion between PS and PVA. In addition, the adsorption of PS to PVA was also improved. Nano indenter measurement indicated the interfacial interaction between PS film and PVA film was obviously enhanced by $40 \%$ after ozonization at room temperature for $2 \mathrm{~h}$ in acidic condition, which will benefit for fabricating laser inertial confinement fusion container.
\end{abstract}

\section{Introduction}

Carbon and hydrogen element with low atomic number is often served as the hollow microspheres material of the container for the laser inertial confinement fusion (ICF) experiment [1-5]. Polystyrene (PS) is an ideal candidate material because of the easy fabricating process of its microspheres. However, the hydrogen barrier property of PS is very poor [6]. Therefore, a barrier layer should be added on the surface of PS to prevent the leakage of fuel gas from the microspheres. Polyacrylonitrile (PAN), Polyvinyl Alcohol (PVA), and carboxymethyl cellulose have a good barrier effect on hydrogen. Among these polymers, only PVA is easy to form microspheres and has been investigated extensively. The diameter of PS-PVA dual hollow microspheres should range from $200 \mu \mathrm{m}$ to $500 \mu \mathrm{m}$ [7].

The shear force of outer sphere is the main factor which can affect the deformation of the microspheres during the preparation of the dual hollow microspheres. The surface tension of droplet is the driving force to maintain its spherical shape [8-11]. The droplets will divide into several smaller droplets if the deformation ratio is bigger than 0.5. However, the exfoliation of PVA shell from PS sphere will occur when the deformation ratio of outer PVA shell exceed a critical value. Two reasons will lead to this unexpected phenomenon, one is the great modulus differences and the other is the weak interaction between PVA and PS. Consequently, the PS spheres will be dissolved by the outer oil phase [12].

The Cox [13] equation for the deformation of liquid drip can be simplified as follows:

$$
D=\frac{1}{2} \cdot\left(1+\frac{3}{16 / \lambda+16}\right) \cdot\left(\frac{1}{\sqrt{(19 \lambda / 40)^{2}+1 / K^{2}}}\right)
$$

where $K=\eta_{m} \dot{\gamma} d / v, \eta_{m}$ is the viscosity of medium, and $\dot{\gamma}$ is the shear rate and $v$ is the surface tension. Here, $\lambda=\eta_{d} / \eta_{m}$, $\eta_{d}$, and $\mathrm{d}$ are the viscosity and diameter of liquid droplets, respectively.

According to (1), the deformation (D) of liquid droplets will increase with its diameter, which will lead to the severe exfoliation between PVA shell and PS sphere for the large hollow sphere. Therefore, it is very difficult to fabricate the large hollow microspheres via the microemulsification technology when the diameter exceeds $500 \mu \mathrm{m}$ [12].

However, the development of ICF experiment requires the hollow microspheres with larger diameter. In this paper, the ozonization technique is introduced to solve this problem. The ozonization technique is often used in polymer 
surface modification for its easy procedure and low cost [1420]. Once polymer is exposed in ozone atmosphere, some carbonyl groups and carboxyl groups will appear on the polymer chains. In addition, some peroxide groups will also generate. The peroxide group will change to hydroxyl group after ultraviolet illumination or heated. Consequently, the surface of polymer will become hydrophilic to some extent $[19,21-23]$. Then the interfacial interaction between PS layer and PVA layer will be enhanced. This technology is a feasible method to avoid the exfoliation between PVA shell and PS sphere for the large hollow sphere.

\section{Experimental Section}

2.1. Materials. Polystyrene (PS) and Polyvinyl Alcohol (PVA) are supplied by J\&K Chemical Company. The number average molecular weight is 250, 000 for PS and 124,000-186,000 for PVA, respectively. All other reagents and solvents were analytical grade products and used without further purification.

\subsection{The Surface Modification of PS Film and the Fabrication of} PS-PVA Bilayer Film. The procedures of surface modification for PS film were conducted as follows. PS was first dissolved in toluene solvent with a proper concentration at $10 \mathrm{wt} \%$. Then the PS solution was spin coated on a silicon substrate and the toluene solvent was removed. The ozone treatment experiment was firstly conducted by dissolving ozone in water at $25^{\circ} \mathrm{C}$. The $\mathrm{pH}$ value was kept at 2 and the concentration of ozone is $20 \mathrm{wt} \%$. The ozone was obtained by an ozone generator. The PS film was immersed in water and its surface was ozonized in succession. Different ozonization time on PS film was carried out.

The PS-PVA bilayer film can be fabricated by spin coating PVA solution on the PS film. PVA was dissolved in water and the weight concentration of PVA solution is $4 \mathrm{wt} \%$. In addition, the thickness of PVA and PS layer is controlled within $15 \mu \mathrm{m}$. The PS-PVA bilayer microsphere was fabricated by using microemulsification technology. Typically, PS solution was dripped into PVA solution together with rotational movement. Finally, PS-PVA bilayer microspheres were obtained after the removal of solvents.

2.3. Characterization. Infrared spectra were measured by using a Spectrum100 spectrometer (PerkinElmer Corporation, USA) with a transmission mode. Ultraviolet-visible (UV-vis) absorption spectra were recorded on a Lambda 900 (PerkinElmer Corporation, USA) spectrometer. The gel permeation chromatography (GPC) measurement was conducted in tetrahydrofuran solvent (WATERS1515, USA). Contact angle of PS films treated with ozone for different time was carried out by a JC2000X (China) machine in water medium. The interaction between PS and PVA was characterized by a Hysitron Triboindenter nano indenter (China). The morphologies of sample were observed by using a metalloscope optical microscopy (CANY C2003B, China). The morphologies of film were sputter-coated with gold and observed by using a JSM-T300 (Japan) field emission scanning electron microscopy with an accelerating voltage

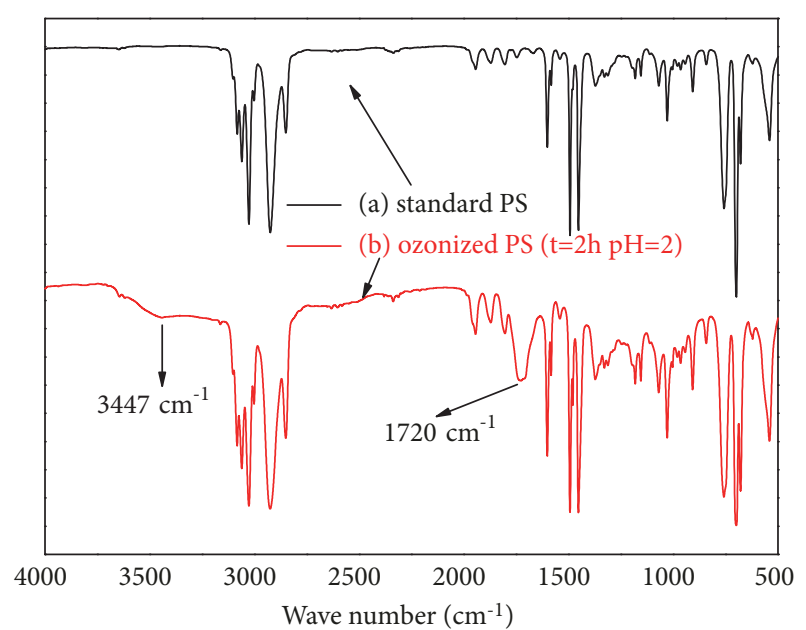

FIGURE 1: FTIR spectra of PS films. (a) PS film ozonized at $\mathrm{PH}=2$, $\mathrm{t}=2 \mathrm{~h}$, and (b) standard PS film.

of $30 \mathrm{kV}$. A Veeco WYKONT1100 (USA) white light interferometer was used to obtain the surface shape of PS film with different treatment.

The adsorption of PVA onto PS film was calculated at room temperature as follows. First, several standard PVA solutions was prepared and the neat PS film and ozonized PS films with different ozonization time were immersed in PVA solution. The adsorption effect of PVA onto PS film can be compared by using Ultraviolet-visible (UV-vis) absorption spectra. To quantify the effect of ozonization time on the adsorption of PVA onto PS film, the adsorption ratio of PVA for neat PS can be defined as 0 when the adsorption time is 0 min. So the adsorption ratio of PVA for ozonized PS can be calculated by comparing the absorption intensity of the neat PS and ozonized PS at different adsorbing time. The adsorption ratio (A) of PVA for ozonized PS with different ozonization time can be calculated by using

$$
A=\frac{I_{n}-I_{o}}{I_{n}} \times 100 \%
$$

where $I_{\mathrm{n}}$ is the absorption intensity of PVA for neat PS and $I_{\mathrm{o}}$ is the absorption intensity of PVA for ozonized PS with different ozonization time.

The morphology of PS-PVA bilayer microspheres was observed by using a microscopy (VHX-600E, KEYENCE, Japan). First, the PS-PVA bilayer microspheres with large diameter (larger than $500 \mu \mathrm{m}$ ) were filtered by using a sieve. The diameter and the thickness of PS layer and PVA layer were measured by an X-ray detector (TXF5011, USA).

\section{Results and Discussion}

3.1. IR Analysis. The infrared spectra characterization of PS film before and after treated by ozone is shown in Figure 1. Compared with the original PS film, the surface modification brings about some different in its absorption peaks when the treated time is $2 \mathrm{~h}$. Two obvious absorption peaks at $1720 \mathrm{~cm}^{-1}$ and $3447 \mathrm{~cm}^{-1}$ are observed clearly, which should 


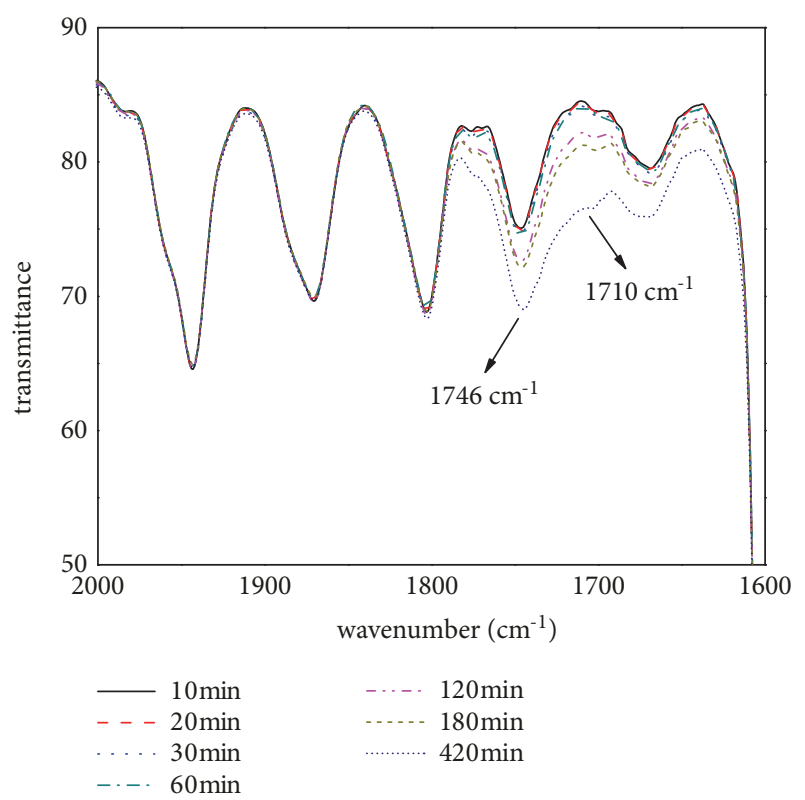

Figure 2: Change of absorption peak at $1720 \mathrm{~cm}^{-1}$ with time on a certain condition $(\mathrm{PH}=2, \mathrm{~T}=323 \mathrm{~K})$.

be ascribed to the stretching vibration of carbonyl groups and hydroxyl groups, respectively. By carefully analyzing the changes of carbonyl groups, the absorption intensity of carbonyl groups is enhanced with modification time in Figure 2. The absorption peak near $1720 \mathrm{~cm}^{-1}$ is further analyzed by double integration and nonlinear fitting, and two new weak absorption peaks $\left(1710 \mathrm{~cm}^{-1}\right.$ and $\left.1746 \mathrm{~cm}^{-1}\right)$ can be found. The possible reason is that the few ketone carbonyl groups exist on the modified PS surface while the carboxyl groups are still paly a dominated role. This phenomenon is well correlated with Liu's report [24]. Therefore, the chemical groups on the ozonization modified PS surface are carboxyl groups and few ketone carbonyl groups.

The amount of carbonyl and hydroxyl groups with ozonization time on the PS film is measured by calculating the area of absorbing peaks. The detailed date of these two functional groups at $323 \mathrm{~K}$ is presented in Figure 3. It can be clearly seen that the amount of both carbonyl and hydroxyl groups are increased with ozonization time. However, the increase tendency is different for the two groups. Obviously, the amount of the carbonyl groups is higher than that of the hydroxyl groups. The possible reason for the increase of these two groups with ozonization time is given as follows.

The ozonization course $[25,26]$ can be described as

$$
\begin{aligned}
\mathrm{O}_{3}(\mathrm{~S}) & \longrightarrow \mathrm{O}_{3}(\mathrm{~T}) \longrightarrow \mathrm{O}_{2}(1 \triangle)+\mathrm{O}\left({ }^{3} \mathrm{P}\right) \\
& \longrightarrow \mathrm{O}_{2}+\mathrm{O}\left({ }^{1} \mathrm{D}\right)
\end{aligned}
$$

The singlet ozone $(\mathrm{O} 3(\mathrm{~S}))$ is a stable substance, while the triplet ozone $(\mathrm{O} 3(\mathrm{~T}))$ is very unstable. Therefore, it is very easy for $\mathrm{O} 3(\mathrm{~T})$ to dissociate to the excited molecule oxygen gas $(\mathrm{O} 2(1 \Delta))$ and the triplet-state oxygen atom $(\mathrm{O}(3 \mathrm{P}))$, respectively; and the $\mathrm{O}(3 \mathrm{P})$ will further transform to the excited $\mathrm{O}(\mathrm{1D})$. The singlet state oxygen atom (1D) can

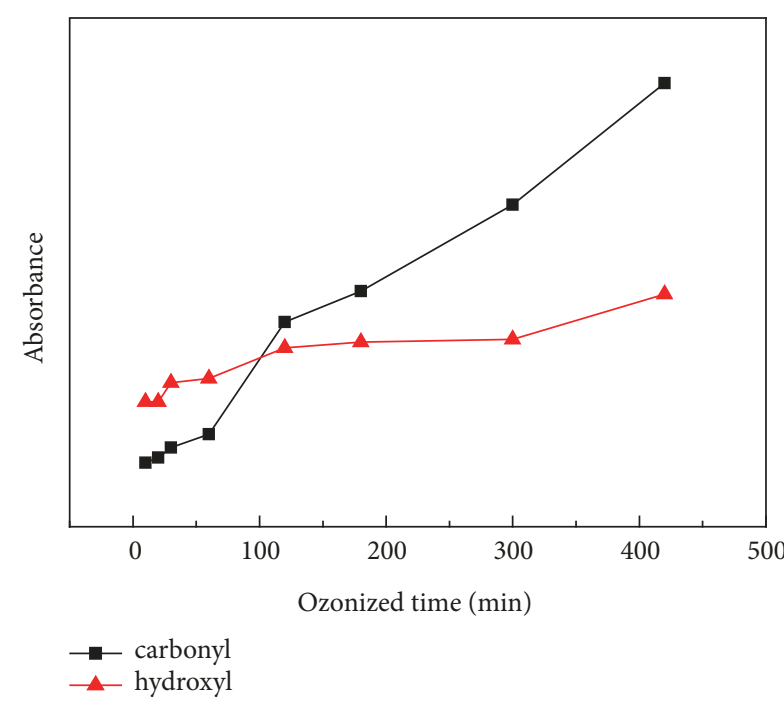

FIGURE 3: Increase of carbonyl content and hydroxyl content at the different ozonized time.

react with PS macromolecular chains through a concerted insertion reaction into $\mathrm{C}-\mathrm{H}$ and $\mathrm{C}-\mathrm{C}$ bonds to form $\mathrm{C}-\mathrm{OR}$ groups, while oxygen (3P) will react with molecular oxygen, ozone, and other radicals through abstracting hydrogen from the polymer to leave a carbon radical, leading to generate more highly oxidized functionalities such as carbonyl and carboxyl groups [27]. By continue increasing the ozonization time, the amount of carbonyl and hydroxyl groups will further increase. Consequently, the interaction between the ozonized PS and the PVA will be enhanced because both of them contain similar polar groups, and this interaction will be further enhanced by increasing the ozonization time. However, excessive ozonization time will lead to several undesirable outcomes. One is the final physical properties of ozonized PS, it will be impaired by introducing too much carbonyl and hydroxyl groups, the other is containing too much carbonyl and hydroxyl groups and it will lead to the adhesion of PS microspheres by hydrogen bond interaction. Therefore, the proper ozonization time is $2 \mathrm{~h}$ by taking the amount of carbonyl and hydroxyl groups into consideration.

3.2. GPC Measurement. To investigate the effect of surrounding conditions on the ozonization PS, series comparisons are carried out by gel permeation chromatography in two different $\mathrm{pH}$ values. The two different $\mathrm{pH}$ values are 2 and 12 . As shown in Figure 4, the weight-average molecular weight (Mw) of PS in two different $\mathrm{pH}$ conditions is calculated. It can be seen that the Mw of ozonized PS is different in the acidic and alkaline treating condition, especially at higher ozonization temperature. Compared with the standard PS sample, little difference is found for the Mw of ozonized PS in acidic surrounding $(\mathrm{pH}<7)$. In contrast, the degradation reaction will generate when the solution condition is alkaline; in other words, the alkaline treating condition will lead to the degradation reaction. The possible reason is that ozone molecular can react with $\mathrm{OH}$ - in solution to produce $\mathrm{OH}_{2}^{-}$, 
TABLE 1: Interaction force between PVA and PS.

\begin{tabular}{lcccc}
\hline PS-PVA film & Transverse crushing load & Friction coefficient & Axial load & Indentation depth \\
\hline Untreated & $1000 \mu \mathrm{N}$ & 0.32 & $3000 \mu \mathrm{N}$ & $890 \mathrm{~nm}$ \\
Ozonized & $1400 \mu \mathrm{N}$ & 0.38 & $3800 \mu \mathrm{N}$ & $890 \mathrm{~nm}$ \\
\hline
\end{tabular}

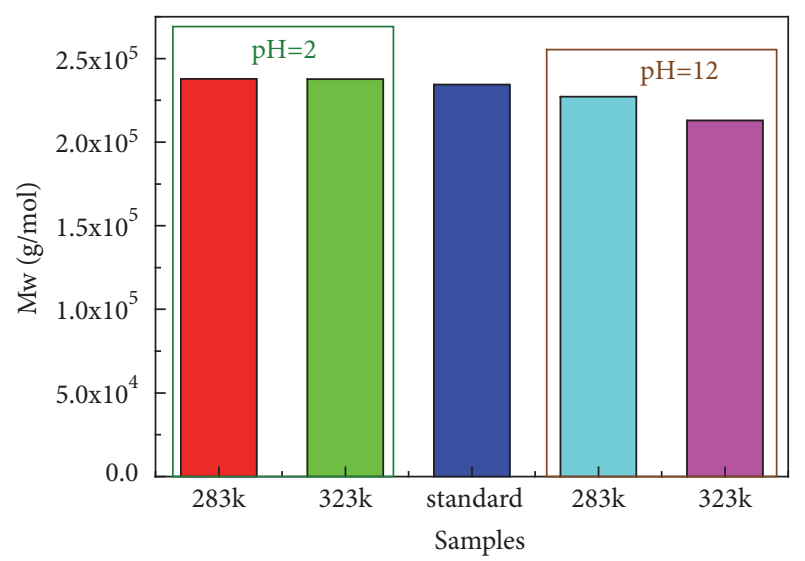

Figure 4: The molecular weight value of the treated PS at the different conditions.

which can induce $\mathrm{OH} \cdot$ radical. The $\mathrm{OH} \bullet$ radical has a high oxidation potential value of $2.80 \mathrm{eV}$. This value is $35 \%$ higher than that of ozone molecular, so $\mathrm{OH}$ - radical can lead to the broken of C-C for PS and further generating water and carbon dioxide [24]. Therefore, the proper treating condition of PS is in acidic condition.

3.3. Contact Angle and UV-vis Characterization. The contact angle of a liquid on a solid surface depends on the physical and chemical properties of the surface, such as wettability, hydrophilicity, and roughness. Thus, measurement of the contact angle on treated PS films gives information on the surface properties. The contact angle for different ozonization PS film to PVA is recorded to study the adsorption properties of PS to PVA; results are presented in Figure 5(a). Figure 5(b) is the adsorption ratio of PS film to PVA for different ozonization time. The ozonization modification technique of PS film is at $323 \mathrm{~K}$ and in acidic condition $(\mathrm{pH}=2)$. The PVA adsorption ratio of ozonized PS with different ozonization time is calculated by using equation (2) and the effect of ozonization time on the adsorption ratio of ozonized PS is shown in Figure 5(b). It can be clearly seen that the adsorption rate of neat PS film is 0 when the adsorbing time is $0 \mathrm{~min}$ and little difference is found by further increasing the adsorbing time. However, an obvious increase of adsorption ratio is found for the ozonized PS and this value will increase with adsorbing time, implying the enhancement of interfacial interaction between PS and PVA by ozone treatment.

Before ozonization modification, the contact angle of PS to PVA is $98.5^{\circ}$. After ozonization for $1 \mathrm{~h}$, this contact angle decreases to $82^{\circ}$, while the adsorption rate of PS to PVA is enhanced. Those phenomena revealed that the surface of PS film has turned from hydrophobic to hydrophilic, which is in agreement with Dengteng Ge [28]. The reason is that the surfaces of ozonization PS film generate carbonyl and hydroxyl groups, which will have strong effect with the hydroxyl groups of PVA by hydrogen bonding [7]. Consequently, the adsorption rate will be enhanced.

Obviously, the contact angle of PS to PVA decreased with the ozonization time; and the adsorption rate of PVA gradually increased. This phenomenon is well correlated with the IR results which demonstrated that the amount of carbonyl and hydroxyl groups is increasing with the ozonization time. The generation of these functional groups can enhance the interfacial interaction between PS and PVA.

3.4. The Toughening Effect of Ozonization PS and the Interfacial Interaction between PS and PVA. The polarizing optical microscopy observation of surface morphologies for PS film before and after ozonization is shown in Figure 6. Half side of the PS film was protected to avoid the ozonization while the other half side was treated by ozone; then the film was immersed into water at $10^{\circ} \mathrm{C}$ to compare their different stress cracking behaviors. After few hours, the half side of the film without ozonization modification began to exhibit cracks. While the other half side of the film began to exhibit cracks after 3 days. There is an obvious difference for the cracks morphologies among the two sides. The shape of the cracks is dendritic and spherical in the left and right side, respectively. These two different cracks are corresponded to brittle and ductile fracture. Therefore, the ozonization modification can result in the toughness of PS film also can stop the development of cracks.

A nano indenter experiment [29] was conducted to characterize the interfacial strength between PS and PVA layers; each layer is $800 \mathrm{~nm}$. The effect of ozonization modification of PS on the interfacial between PS and PVA was analyzed. When a critical loading is carried; a sudden change of friction coefficient will appear. Therefore, the sudden change of friction coefficient can be used to judge the interfacial debonding of PS and PVA. The calculated data of two different systems was listed in Table 1; one is for the untreated PS coated PVA and the other is for the ozonization PS and PVA (the ozonization condition is at room temperature for $2 \mathrm{~h}$ in acidic condition, $\mathrm{pH}=2$ ). Obviously, the critical loading of layers between ozonized PS and PVA is increased by $40 \%$ compared with that of untreated PS and PVA, which indicates that the interfacial interaction between ozonized PS and PVA is enhanced. This result is well complied with the increase adsorption between PS and PVA in Figure 5(b).

\subsection{The Effect of Ozonization on the Surface Morphologies} of PS Film. The effect of ozonization time on the surface morphologies of PS film is shown in Figure 7. Compared with the smooth surface of the untreated PS, some concaves were 


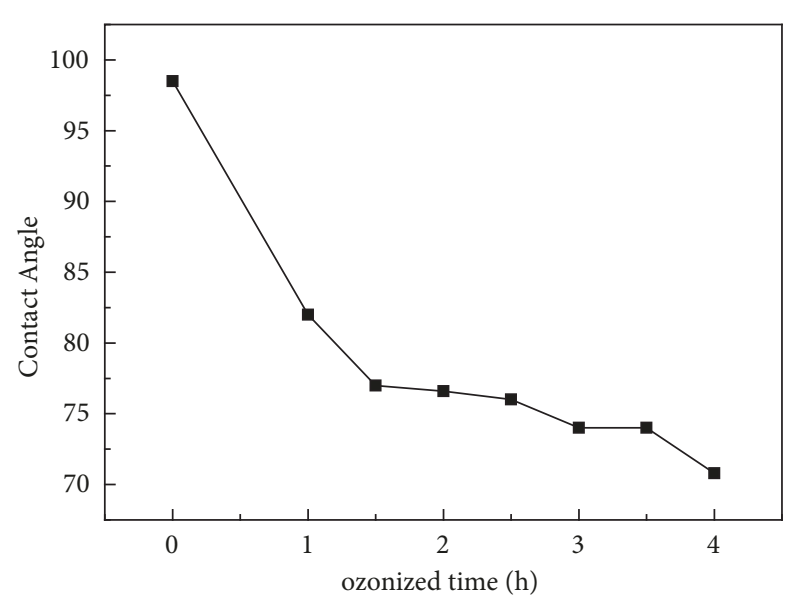

(a) Contact angle of surfactant functionalized PS films with different ozonized time

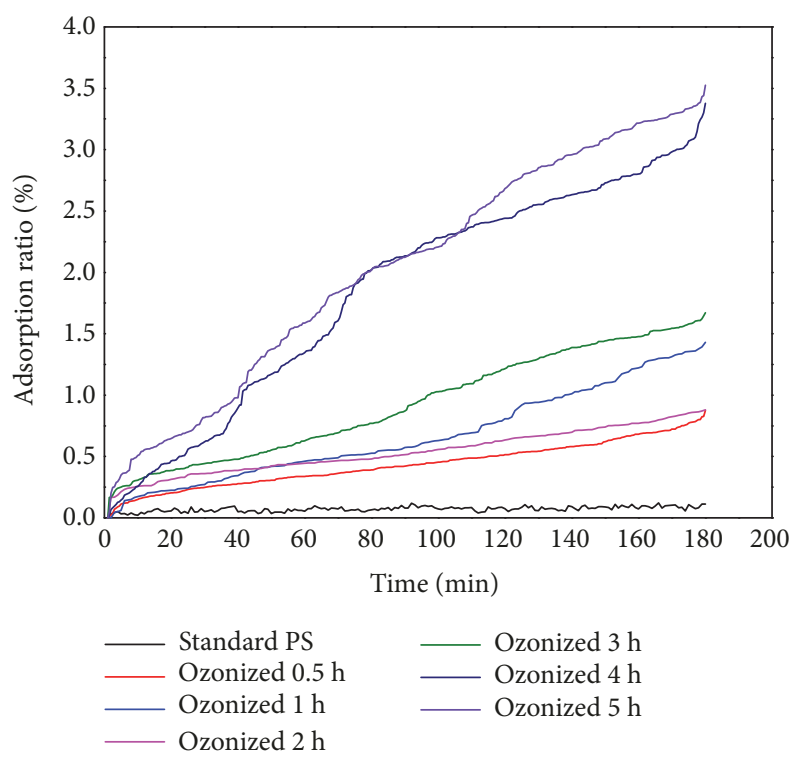

(b) PVA adsorption for ozonated PS films with different ozonized time

FIGURE 5: Contact angle and PVA adsorption of ozonated PS films with different ozonized time.
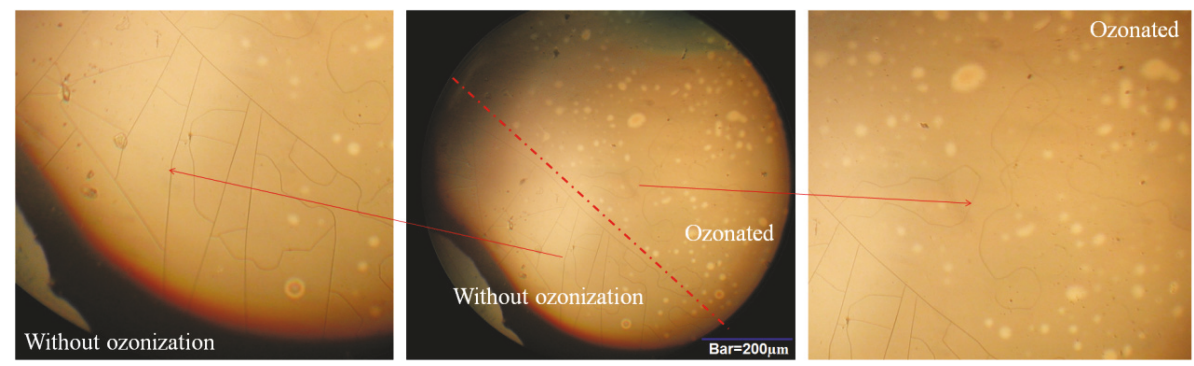

FIGURE 6: Pictures of the surface of PS film before and after ozonization.

found on the surface of PS film after ozonization at $323 \mathrm{~K}$. These concaves resulted from the chemical reaction between PS chains and ozone [30-33]. The average diameter of concaves is about $3 \mu \mathrm{m}$ and the amount of concaves increases with the ozonization time. The existence of these concaves can benefit the enhancement of the interfacial interaction between PS and PVA by providing large anchoring spots. Figure 8 is the thickness map collected from the white light interferometer for the ozonized PS film. The ozonization time is $2 \mathrm{~h}$ and the $\mathrm{pH}$ value is 2 . It can be clearly seen that the surface of ozonized PS film is not very coarse and a few defects are found on this surface. However, serious problem will be brought about when the ozonization time is as long as $8 \mathrm{~h}$ because too many concaves will deteriorate the mechanical properties of PS film.

It is known that the survival rate of PS-PVA hollow microsphere with large diameter will be restrained due to the large deformation of each layer on the basis of Cox equation. Therefore, the interaction between PS and PVA layer should be enhanced to fabricate large hollow microsphere with excellent stability. The enhancement of the interaction between PS and PVA through ozone treatment will lead to the increasing of stability for PS-PVA bilayer microspheres with large dimension (the diameter is larger than $500 \mu \mathrm{m}$ ). Figure 9 is the pictures of the bilayer PS-PVA microspheres treated by ozone. The thickness of each layer and the survival rate of PS-PVA hollow microsphere with large diameter are listed in Tables 2 and 3, respectively. The average diameter of PS-PVA bilayer microspheres can reach to $778 \mu \mathrm{m}$. After ozone treatment, the survival rate of PS-PVA hollow microsphere with large diameter is increased to $50 \%$, a remarkable enhancement compared with the untreated PSPVA one.

\section{Conclusion}

The ozonization modification method was conducted to the PS film to enhance the interfacial interaction between PS and PVA in this paper. The experimental results revealed that the carbonyl and hydroxyl groups were introduced to the surface of PS and the amount of functional groups increased with the ozonization time. The proper modification condition is in acidic condition. After ozonization modification, the hydrophilic PS was improved and the adsorption of PS to 
TABLE 2: Parameters of the PS-PVA bilayer hollow microspheres.

\begin{tabular}{lccc}
\hline $\begin{array}{l}\text { Diameter of PS-PVA } \\
(\mu \mathrm{m})\end{array}$ & $\begin{array}{c}\text { Thickness of PS-PVA } \\
\text { bilayer }(\mu \mathrm{m})\end{array}$ & $\begin{array}{c}\text { Thickness of PVA } \\
(\mu \mathrm{m})\end{array}$ & Thickness of PS $(\mu \mathrm{m})$ \\
\hline 778.35 & 13.64 & 3.26 & 10.38 \\
\hline
\end{tabular}

TABLE 3: Survival rate of PS-PVA hollow microspheres of large diameter (larger than $500 \mu \mathrm{m}$ ).

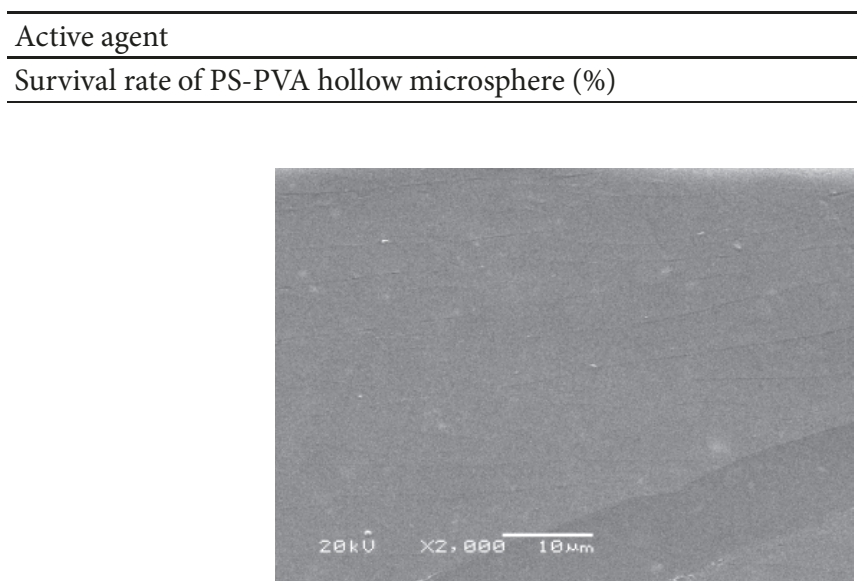

(a) Standard PS film

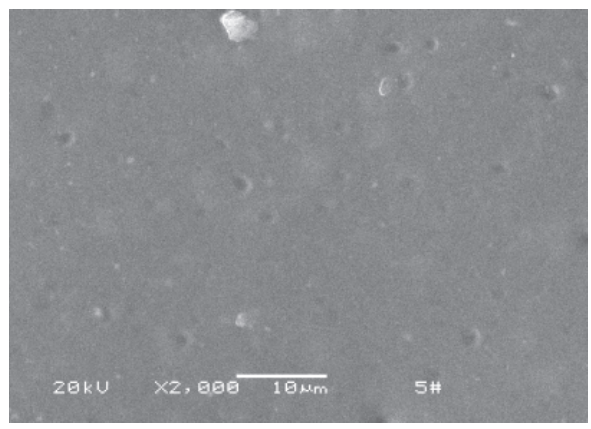

(c) $\mathrm{pH}=2, \mathrm{~T}=323 \mathrm{~K}, 2 \mathrm{~h}$

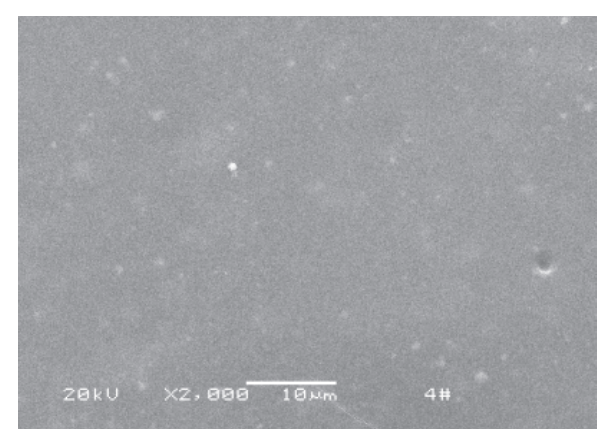

(b) $\mathrm{pH}=2, \mathrm{~T}=323 \mathrm{~K}, 1 \mathrm{~h}$

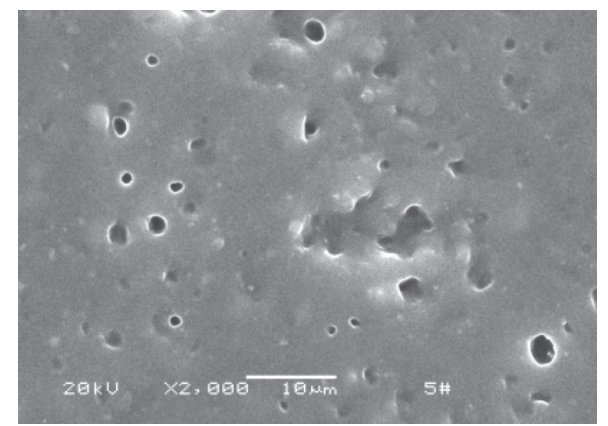

(d) $\mathrm{pH}=2, \mathrm{~T}=323 \mathrm{~K}, 8 \mathrm{~h}$

FIgURE 7: The SEM pictures of the surface of PS film at the different condition.

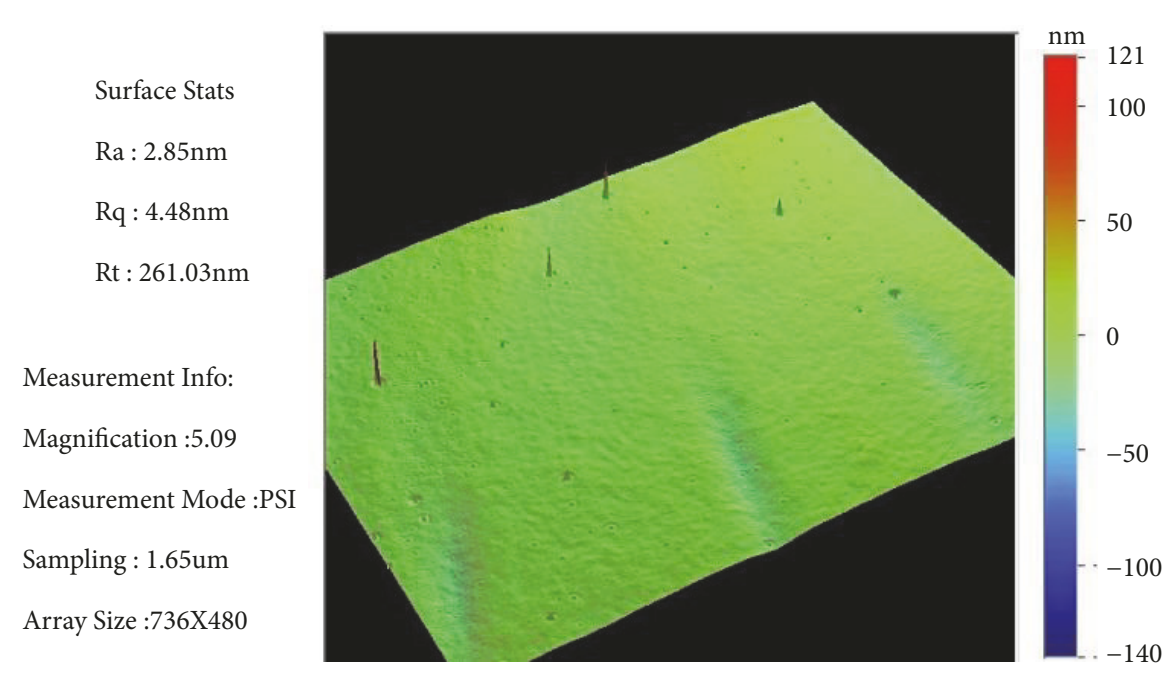

FIGURE 8: The pictures of the surface of PS film after ozonization at $\mathrm{pH}=2, \mathrm{~T}=323 \mathrm{~K}$, and $\mathrm{t}=2 \mathrm{~h}$. 


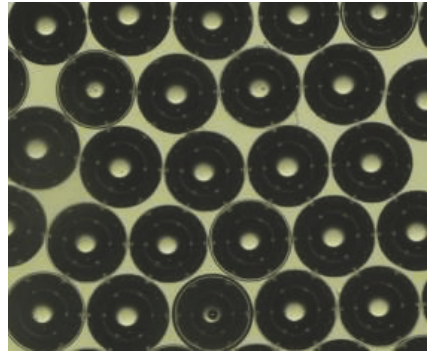

(a)

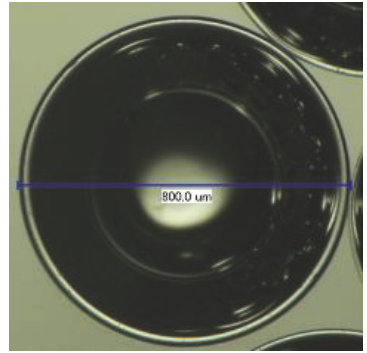

(b)

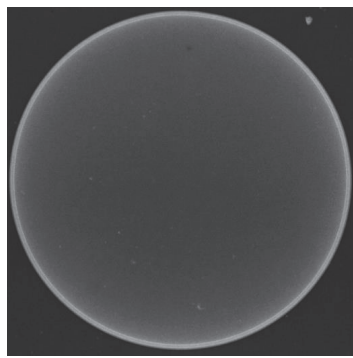

(c)

FIgURE 9: The microscope and X-ray pictures of PS-PVA bilayer hollow microspheres (a) ((b) is the picture with magnification of 200 for (a) and (c) is the X-ray picture of (a)).

PVA was also enhanced. The introduction of the hydrophilic groups could lead to the enhancement interfacial interaction between PS and PVA film. Therefore, the interfacial debonding of the PS-PVA bilayer hollow microspheres could be avoided by this ozonization technique.

\section{Data Availability}

The data used to support the findings of this study are available from the corresponding author upon request.

\section{Conflicts of Interest}

The authors declare that they have no conflicts of interest.

\section{Acknowledgments}

The authors are grateful to the China Academy of Engineering Physics for financial support (2014B0302052).

\section{References}

[1] M. F. Liu, S. F. Chen, X. B. Qi et al., "Improvement of wall thickness uniformity of thick-walled polystyrene shells by density matching," Chemical Engineering Journal, vol. 241, pp. 466-476, 2014.

[2] J. Li, L. Su, M. F. Liu et al., "Influence of sucrose on the stability of W1/O/W2 double emulsion droplets," RSC Advances, vol. 5, p. 83089, 2015.

[3] X. J. Zhang, J. Li, K. Cao, Y. Yi, J. X. Yang, and B. Li, "Synthesis and characterization of $\mathrm{B}-\mathrm{C}$ polymer hollow microspheres from a new organodecaborane preceramic polymer," RSC Advances, vol. 5, p. 86214, 2015.

[4] R. R. Paguio, C. A. Frederick, J. F. Hund et al., "Fabrication capabilities for spherical foam targets used in ICF experiments," in Proceedings of the 17th Target Fabrication Specialist Meeting, San Diego, CA, USA, 2006.

[5] L. Su, J. Li, J. Li et al., "Influence of ionic surface active agent on distribution properties of PS-PVA double emulsion," High Power Laser and Particle Beams, vol. 26, no. 2, Article ID 022012(5), 2014.

[6] Y. Honarpazhouh, F. R. Astaraei, H. R. Naderi, and O. Tavakoli, "Electrochemical hydrogen storage in Pd-coated porous silicon/graphene oxide," International Journal of Hydrogen Energy, vol. 41, no. 28, pp. 12175-12182, 2016.
[7] L. Su, J. Li, Q. M. Chu et al., "Effect of Tween 20 nonionic surfactant on preparation of large size double-layer hollow microspheres," High Power Laser and Particle Beams, vol. 27, no. 12, Article ID 27122007, 2015.

[8] Q. Chen, S. F. Chen, M. F. Liu et al., "Influence of fluorobenzene mass transfer on the qualities of poly- $\alpha$-methylstyrene shells," RSC Advances, vol. 8, pp. 3687-3693, 2018.

[9] M. F. Liu, Y. Q. Zheng, J. Li et al., "Effects of molecular weight of PVA on formation, stability and deformation of compound droplets for ICF polymer shells," Nuclear Fusion, vol. 57, no. 1, Article ID 016018, 2017.

[10] S. Tasoglu, G. Kaynak, A. J. Szeri, U. Demirci, and M. Muradoglu, "Impact of a compound droplet on a flat surface: a model for single cell epitaxy," Physics of Fluids, vol. 22, no. 8, Article ID 082103, 2010.

[11] P. Gao and J. J. Feng, "Spreading and breakup of a compound drop on a partially wetting substrate," Journal of Fluid Mechanics, vol. 682, pp. 415-433, 2011.

[12] L. Su, S. F. Chen, M. F. Liu et al., "Research progress of fabricating polyvinyl alcohol coating on plastic microsphere," High Power Laser and Particle Beams, vol. 24, no. 7, pp. 15171522, 2012.

[13] R. G. Cox, "The deformation of a drop in a general timedependent fluid flow," Journal of Fluid Mechanics, vol. 37, no. 3, pp. 601-623, 1969.

[14] T. N. Murakami, Y. Fukushima, Y. Hirano, Y. Tokuoka, M. Takahashi, and N. Kawashima, "Modification of PS films by combined treatment of ozone aeration and UV irradiation in aqueous ammonia solution for the introduction of amine and amide groups on their surface," Applied Surface Science, vol. 249, p. $425,2005$.

[15] L. F. Macmanus, M. J. Walzak, and N. S. Mcintyre, "Study of ultraviolet light and ozone surface modification of polypropylene," Journal of Polymer Science Part A: Polymer Chemistry, vol. 37, no. 14, p. 2489, 1999.

[16] E. Harel, S. E. Meltzer, A. A. G. Requicha, M. E. Thompson, and B. E. Koel, "Fabrication of polystyrene latex nanostructures by nanomanipulation and thermal processing," Nano Letters, vol. 5, no. 12, p. 2624, 2005.

[17] L. Wang, S. B. Gao, J. J. Wang, W. C. Wang, L. Q. Zhang, and M. Tian, "Surface modification of UHMWPE fibers by ozone treatment and UV grafting for adhesion improvement," The Journal of Adhesion, vol. 94, no. 1, pp. 30-45, 2018.

[18] T. Clark Jr, J. D. Ruiz, H. Fan, C. J. Brinker, B. I. Swanson, and A. N. Parikh, "A new application of UV-ozone treatment in 
the preparation of substrate-supported, mesoporous thin films," Chemistry of Materials, vol. 12, p. 3879, 2000.

[19] M. R. Davidson, S. A. Mitchell, and R. H. Bradley, "UVozone modification of plasma-polymerised acetonitrile films for enhanced cell attachment," Colloids and Surfaces B: Biointerfaces, vol. 34, no. 4, pp. 213-219, 2004.

[20] X. Yu, A. Beharaj, M. W. Grinstaff, and O. K. C. Tsui, "Modulation of the effective viscosity of polymer films by ultraviolet ozone treatment," Polymer Journal, vol. 116, pp. 498-505, 2017.

[21] P. L. Yue, "Modelling of kinetics and reactor for water purification by photo-oxidation," Chemical Engineering Science, vol. 48, no. 1, pp. 1-11, 1993.

[22] T. N. Murakami, Y. Fukushima, Y. Hirano, Y. Tokuoka, M. Takahashi, and N. Kawashima, "Surface modification of polystyrene and poly(methyl methacrylate) by active oxygen treatment," Colloids and Surfaces B: Biointerfaces, vol. 29, no. 2-3, pp. 171179, 2003.

[23] T. N. Murakami, M. Takahashi, and N. Kawashima, "Decomposition of aromatic compounds by active oxygen generator," Chemistry Letters, vol. 29, no. 11, pp. 1312-1313, 2000.

[24] Y. Y. Liu, Q. B. Kan, S. Wei, Z. W. Zhang, and S. F. Chen, "Surface modification of PS films by ozone," High Power Laser and Particle Beams, vol. 23, no. 1, p. 111, 2011.

[25] P. Somathilake, J. A. Dominic, G. Achari, C. H. Langford, and J.-H. Tay, "Degradation of carbamazepine by photo-assisted ozonation: influence of wavelength and intensity of radiation," Ozone: Science \& Engineering, vol. 40, no. 2, pp. 113-121, 2018.

[26] C. Ding, D. K. Yuan, Z. H. Wang et al., "Ozone production influenced by increasing gas pressure in multichannel dielectric barrier discharge for positive and negative pulse modes," Ozone: Science \& Engineering, vol. 40, no. 3, p. 228, 2018.

[27] D. O. H. Teare, C. Ton-That, and R. H. Bradley, "Surface characterization and ageing of ultraviolet-ozone-treated polymers using atomic force microscopy and x-ray photoelectron spectroscopy," Surface and Interface Analysis, vol. 29, no. 4, p. $276,2000$.

[28] D. T. Ge, Y. Li, L. L. Yang, Z. Fan, C. Liu, and X. Zhang, "Improved self-assembly through UV/ozone surfacemodification of colloidal spheres," Thin Solid Films, vol. 519, pp. 5203-5207, 2011.

[29] Z. H. Hodzic, J. Stachurski, and K. Kim, "Nano-indentation of polymer-glass interfaces part I. Experimental and mechanical analysis," Polymer, vol. 41, no. 18, pp. 6895-6905, 2000.

[30] R. Yang, Q. H. He, C. H. Wang, and S. Q. Sun, "Surface modification of polystyrene microsphere using ozone treatment," Ferroelectrics, vol. 530, no. 1, pp. 130-135, 2018.

[31] Z. Yoshimitsu, A. Nakajima, T. Watanabe, and K. Hashimoto, "Effects of surface structure on the hydrophobicity and sliding behavior of water droplets," Langmuir, vol. 18, no. 15, pp. 58185822, 2002.

[32] S. A. Mitchell, A. H. C. Poulsson, M. R. Davidson, N. Emmison, A. G. Shard, and R. H. Bradley, "Cellular attachment and spatial control of cells using micro-patterned ultra-violet/Ozone treatment in serum enriched media," Biomaterials, vol. 25, no. 18, pp. 4079-4086, 2004.

[33] D. O. H. Teare, C. Ton-That, and R. H. Bradley, "Surface characterization and ageing of ultraviolet-ozone-treated polymers using atomic force microscopy and x-ray photoelectron spectroscopy," Surface and Interface Analysis, vol. 29, pp. 276$283,2000$. 


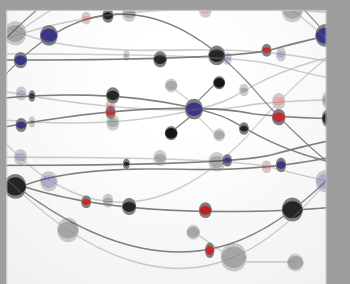

The Scientific World Journal
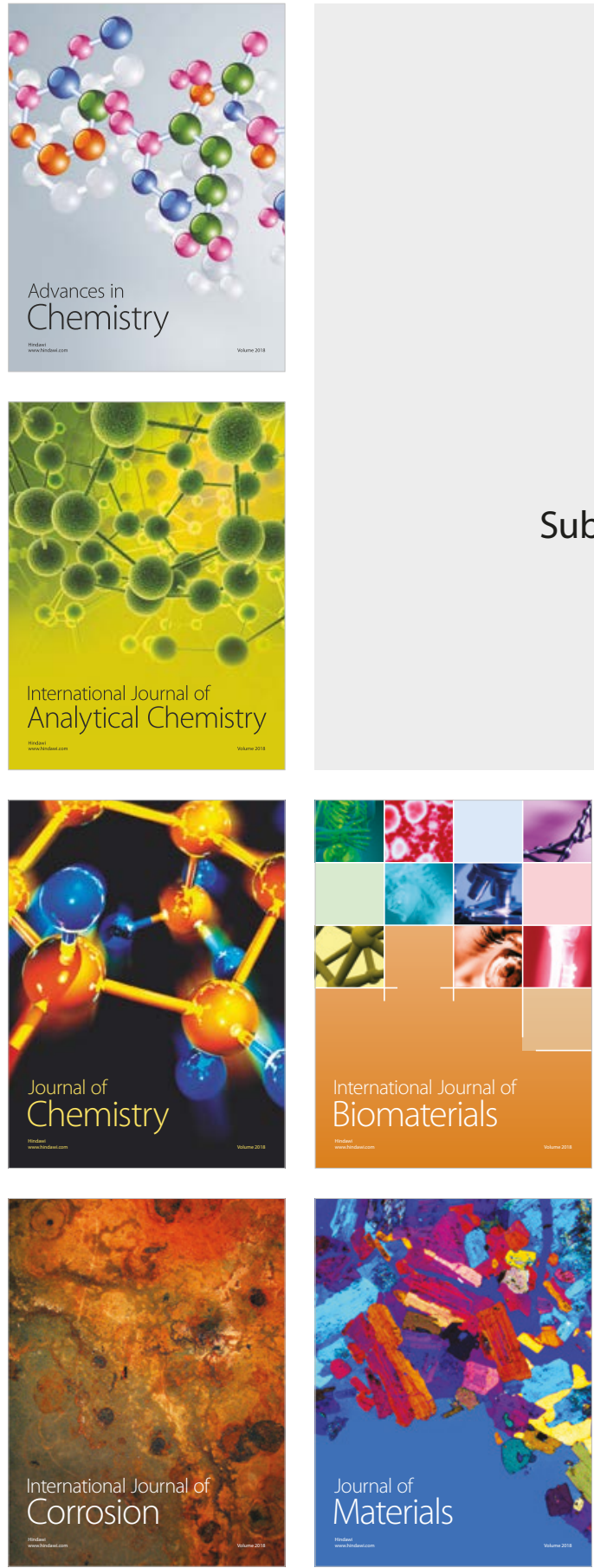

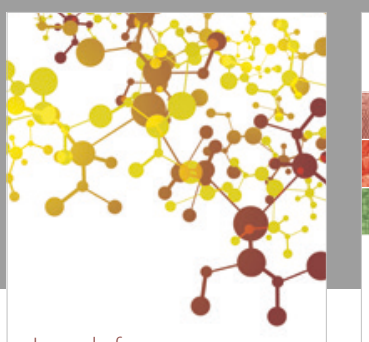

Journal of

Applied Chemistry
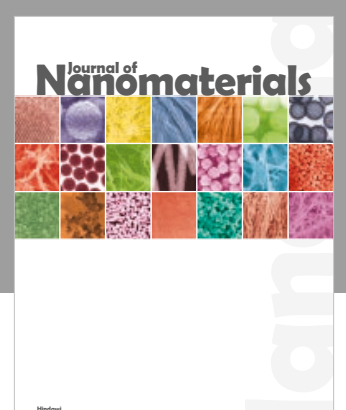

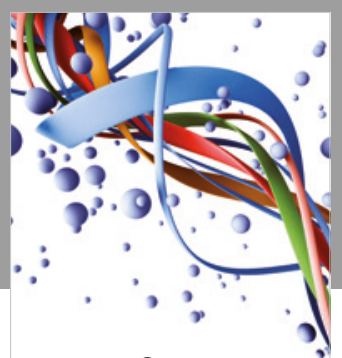

Scientifica

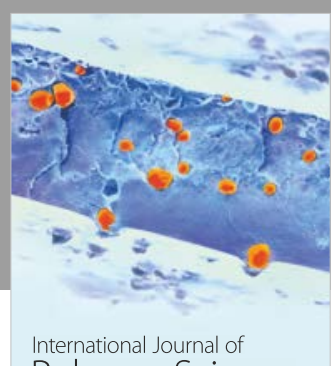

Polymer Science

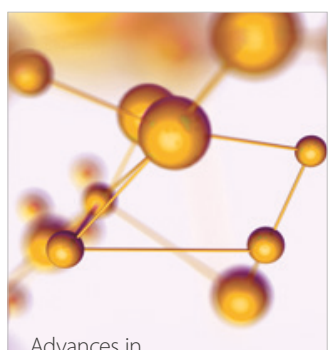

Physical Chemistry
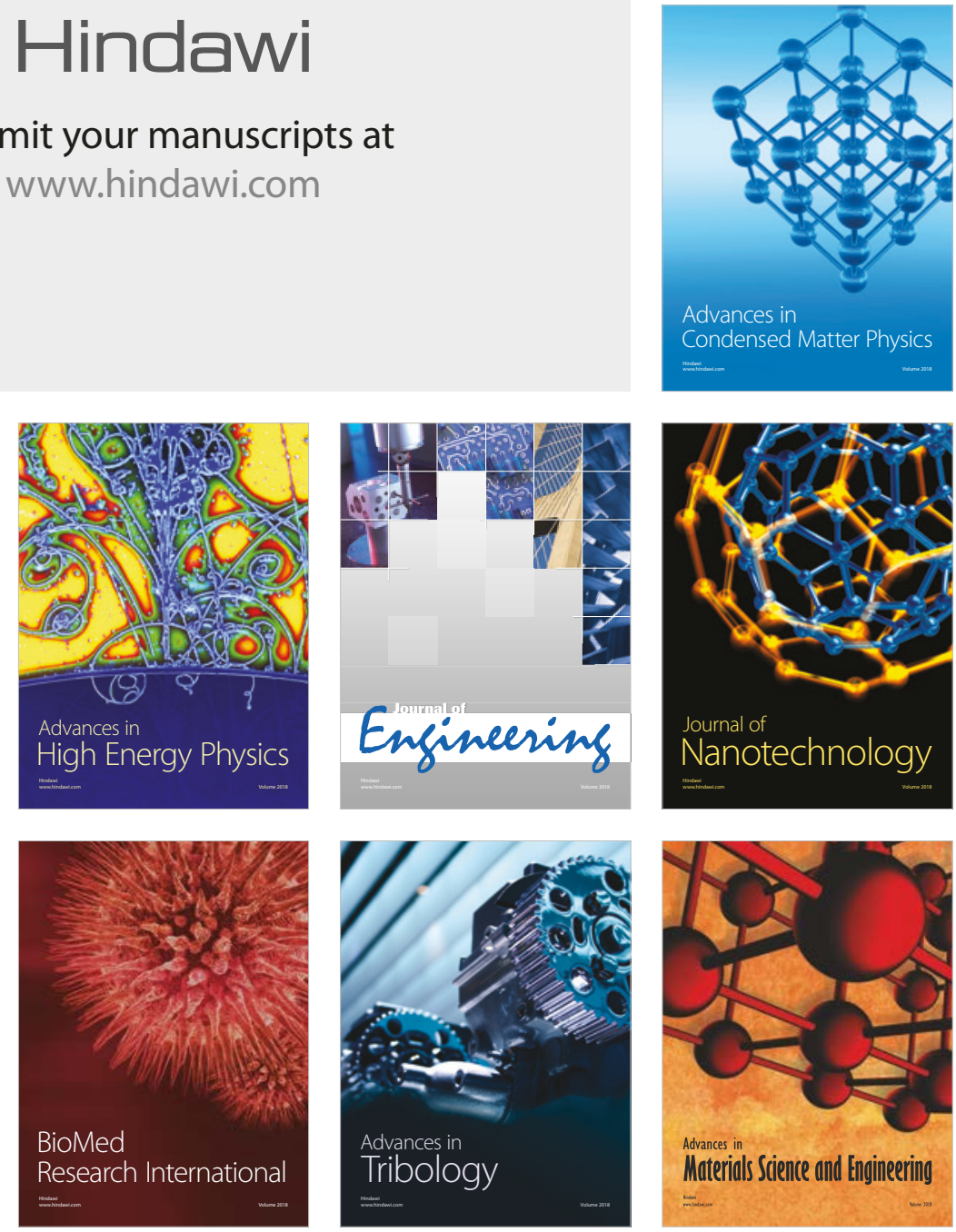